\title{
Uniform hyperbolicity of the curve graph via surgery sequences
}

\author{
MATT Clay \\ KASRA RAFI \\ SAUL SCHLEIMER
}

\begin{abstract}
We prove that the curve graph $\mathcal{C}^{(1)}(S)$ is Gromov-hyperbolic with a constant of hyperbolicity independent of the surface $S$. The proof is based on the proof of hyperbolicity of the free splitting complex by Handel and Mosher, as interpreted by Hilion and Horbez.
\end{abstract}

57M99; 30F60

\section{Introduction}

In recent years the curve graph has emerged as the central object in a variety of areas, such as the study of Kleinian groups (see Minsky [16; 17] and Brock, Canary and Minsky [6]), Teichmüller spaces (see the second author [18; 19] and Brock, Masur and Minsky [7]) and mapping class groups (see Masur and Minsky [15] and Behrstock, Kleiner, Minsky and Mosher [2]). The initial breakthrough was the result of Masur and Minsky showing that the curve graph is Gromov hyperbolic [14].

In this note, we give a new proof of the hyperbolicity of all curve graphs. We improve on the original proof by additionally showing that the hyperbolicity constants are uniform, that is, independent of the topology of the surface.

We use the same hyperbolicity criterion as defined and used by Masur and Minsky [14, Definition 2.2]. Suppose $\mathcal{X}$ is a graph, equipped with a family of paths, and each path $\sigma$ is equipped with a projection map $\pi_{\sigma}: \mathcal{X} \rightarrow \sigma$. If the family of paths and projection maps satisfy the retraction, Lipschitz and contraction axioms, as stated in Section 5, then $\mathcal{X}$ is hyperbolic [14, Theorem 2.3]. We provide a proof of this in Section 6. Bestvina and Feighn recently used a similar argument to show that the free factor graph of a free group is Gromov hyperbolic [3].

For the curve graph and the free factor graph, another, more geometric, space played the key role in the definition of paths and projection maps. For the curve graph this was the 
Teichmüller space; for the free factor graph it was the outer space. An understanding of geodesics in the geometric spaces was necessary to define the family of paths and their projection maps.

The splitting graph, another variant of the curve graph for the free group, was recently shown to be hyperbolic by Handel and Mosher [9]. They also used the hyperbolicity criterion of Masur and Minsky. A novel aspect of their approach was to dispense with the ancillary geometric space; instead they defined projection as if the space were hyperbolic and the family of paths were geodesics. Specifically, given three points $x$, $y$ and $z$ in the space, the projection of $z$ to the path $\sigma$ from $x$ to $y$ is the first point along $\sigma$ that is close (in a uniform sense) to the path from $z$ to $y$. See Figure 1.1.

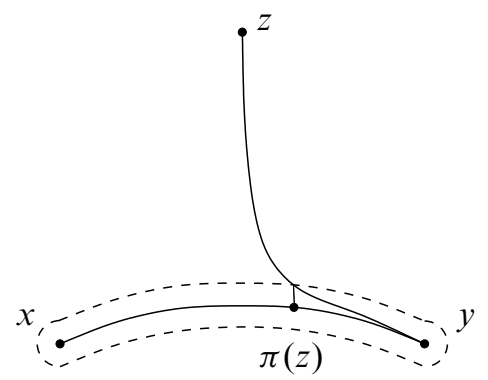

Figure 1.1: Handel-Mosher projection of a point $z$ to the path from $x$ to $y$

The paths used by Handel and Mosher in the splitting graph have a key property that is very reminiscent of negatively curved spaces: exponential divergence. In the other direction we find exponential convergence. On a small scale, Handel and Mosher show paths that start distance two apart, and that have the same target, must "intersect" after a distance depending only on the rank of the free group. On a larger scale, this implies that the "girth" of two paths, with the same target, is cut in half after a similar distance. This property is the main tool used to verify the Masur and Minsky axioms.

Hilion and Horbez [13] gave a geometric spin to Handel and Mosher's argument; this led them to an alternative proof of hyperbolicity of the splitting graph (in their setting called the sphere graph). Their paths were surgery sequences of spheres in the doubled handlebody. We closely follow their setup and use surgery sequences of arcs and curves as defined by Hatcher [11] as paths in the curve graph. We now state our main results. Let $S=S_{g, n}$ be a surface of genus $g$ with $n$ boundary components, let $\mathcal{C}(S)$ be the complex of curves, and let $\mathcal{A C}(S)$ be the complex of curves and arcs; we defer the definitions to Section 2. We add a superscript (1) to denote the one-skeleton.

Theorem 6-5 There is a constant $\mathrm{U}$ such that if $3 g-3+n \geq 2$ and $n>0$ then $\mathcal{A C}^{(1)}\left(S_{g, n}\right)$ is $\mathrm{U}$-hyperbolic. 
The inclusion $\mathcal{C}^{(1)}\left(S_{g, n}\right) \rightarrow \mathcal{A \mathcal { C } ^ { ( 1 ) }}\left(S_{g, n}\right)$ gives a quasi-isometric embedding with constants independent of $g$ and $n$. We deduce the following.

Corollary 7-1 There is a constant $\mathrm{U}$ such that if $3 g-3+n \geq 2$ and $n>0$ then $\mathcal{C}^{(1)}\left(S_{g, n}\right)$ is U-hyperbolic.

We also prove uniform hyperbolicity in the closed case, when $n=0$. This follows from Theorem 6-5, as $\mathcal{C}^{(1)}\left(S_{g, 0}\right)$ isometrically embeds in $\mathcal{C}^{(1)}\left(S_{g, 1}\right)$.

Theorem 7-2 There is a constant $U$ such that if $3 g-3 \geq 2$ then $\mathcal{C}^{(1)}\left(S_{g}\right)$ is Uhyperbolic.

As noted above, the various constants appearing in our argument are uniform. This is mostly due to Lemma 3-3 which shows that paths that start distance two apart, and that have the same target, must "intersect" after a uniform distance.

After the original paper of Masur and Minsky, Bowditch [4] and Hamenstädt [8] also gave proofs of the hyperbolicity of the curve graph. In all of these the upper bound on the hyperbolicity constant depended on the topology of the surface $S$. During the process of writing this paper, several other proofs of uniform hyperbolicity emerged. Bowditch [5] has refined his approach to obtain uniform constants using techniques he developed in [4]; the proof by Aougab [1] has many common themes with the work of Bowditch. The work of Hensel, Przytycki and Webb [12] also uses surgery paths and has other points of contact with our work. However Hensel, Przytycki and Webb do not use the Masur-Minsky criterion; they also obtain much smaller hyperbolicity constants than given here.

Acknowledgements We thank the Centre de Recerca Matemàtica for its hospitality during its 2012 research program on automorphisms of free groups. The first author is partially supported by NSF grant DMS-1006898. The second author is partially supported by NSF grant DMS-1007811. The third author is partially supported by EPSRC grant EP/I028870/1. This work is in the public domain.

\section{Background}

Let $S=S_{g, n}$ be a connected, compact, oriented surface of genus $g$ with $n$ boundary components. We make the standing assumption that the complexity of $S$, namely $3 g-3+n$, is at least two. This rules out three surfaces: $S_{0,4}, S_{1}, S_{1,1}$. In each case the arc and curve complex is a version of the Farey graph; the Farey graph has hyperbolicity constant one when we restrict to the vertices, and $\frac{3}{2}$ when we include the edges. 


\subsection{Arcs and curves}

A properly embedded curve or arc $\alpha \subset S$ is essential if $\alpha$ does not cut a disk off of $S$. A properly embedded curve $\alpha$ is nonperipheral if it does not cut an annulus off of $S$. Define $\mathcal{A C}(S)$ to be the set of ambient isotopy classes of essential arcs and essential nonperipheral curves.

For classes $\alpha, \beta \in \mathcal{A C}(S)$, define the geometric intersection number $\mathrm{i}(\alpha, \beta)$ to be the minimal intersection number among representatives. A nonempty subset $A \subset \mathcal{A C}(S)$ is a system of arcs and curves, or simply a system, if for all $\alpha, \beta \in A$ we have $\mathrm{i}(\alpha, \beta)=0$. We now give $\mathcal{A C}(S)$ the structure of a simplicial complex by taking systems for the simplices. We use $\mathcal{C}(S)$ to denote the subcomplex of $\mathcal{A C}(S)$ spanned by curves alone. Note that these are flag complexes: when the one-skeleton of a simplex is present, so is the simplex itself. Let $\mathcal{K}^{(1)}$ denote the one-skeleton of a simplicial complex $\mathcal{K}$.

If $\alpha$ and $\beta$ are vertices of $\mathcal{A C}(S)$ then we use $d_{S}(\alpha, \beta)$ to denote the combinatorial distance coming from $\mathcal{A C}^{(1)}(S)$. Given two systems $A, B \subset \mathcal{A C}(S)$ we define their outer distance to be

$$
\operatorname{outer}(A, B)=\max \left\{d_{S}(\alpha, \beta) \mid \alpha \in A, \beta \in B\right\}
$$

and their inner distance to be

$$
\operatorname{inner}(A, B)=\min \left\{d_{S}(\alpha, \beta) \mid \alpha \in A, \beta \in B\right\} .
$$

For $\beta \in \mathcal{A C}(S)$ we write inner $(A, \beta)$ instead of $\operatorname{inner}(A,\{\beta\})$, and similarly for the outer distance. If $A$ and $B$ are systems and $C \subset A$ is a subsystem then

$$
\text { inner }(A, B) \leq \operatorname{inner}(C, B) \leq \operatorname{inner}(A, B)+1 \text {. }
$$

For any three systems $A, B$ and $C$, there is a triangle inequality up to an additive error of one, namely

$$
\operatorname{inner}(A, B) \leq \operatorname{inner}(A, C)+\operatorname{inner}(C, B)+1 .
$$

The additive error can be reduced to zero when $C$ is a singleton.

Suppose $A \subset \mathcal{A C}(S)$ is a system and $\gamma \in \mathcal{A C}(S)$ is an arc or curve. We say $\gamma$ cuts $A$ if there is an element $\alpha \in A$ so that $\mathrm{i}(\gamma, \alpha)>0$. If $\gamma$ does not cut $A$ then we say $\gamma$ misses $A$.

A system $A$ fills $S$ if every curve $\gamma \in \mathcal{C}(S)$ cuts $A$. Note that filling systems are necessarily comprised solely of arcs. A filling system $A$ is minimal if no subsystem is filling. The next lemma follows by considering Euler characteristic. 
Lemma 2-3 Suppose $S=S_{g, n}$, with $n>0$, and suppose $A$ is a minimal filling system. If $S-A$ is a disk then $|A|=2 g-1+n$. On the other hand, if $S-A$ is a collection of peripheral annuli then $|A|=2 g-2+n$.

\subsection{Surgery}

If $X$ is a space and $Y \subset X$ is a subspace, let $N=N_{X}(Y)$ denote a small regular neighborhood of $Y$ taken in $X$. Let $\operatorname{fr}(N)=\overline{\partial N-\partial X}$ be the frontier of $N$ in $X$.

Now suppose $A$ is a system and $\omega$ is a directed arc cutting $A$. We seek to describe Hatcher's surgery of $A$ along $\omega$ [11]. Choose representatives to minimize intersection numbers between elements of $A$ and $\omega$. Suppose $\delta$ is the component of $\omega-A$ containing the initial point of $\omega$. Thus $\delta$ meets only one component of $A$, say $\alpha$; we call $\alpha$ the active element of $A$. Let $N=N_{S}(\alpha \cup \delta)$ be a neighborhood. Let $N^{\prime}$ be the component of $N-\alpha$ containing the interior of $\delta$. Let $\alpha^{\omega}$ be the component(s) of $\operatorname{fr}(N)$ that are contained in $N^{\prime}$. See Figure 2.1 for the two possible cases.
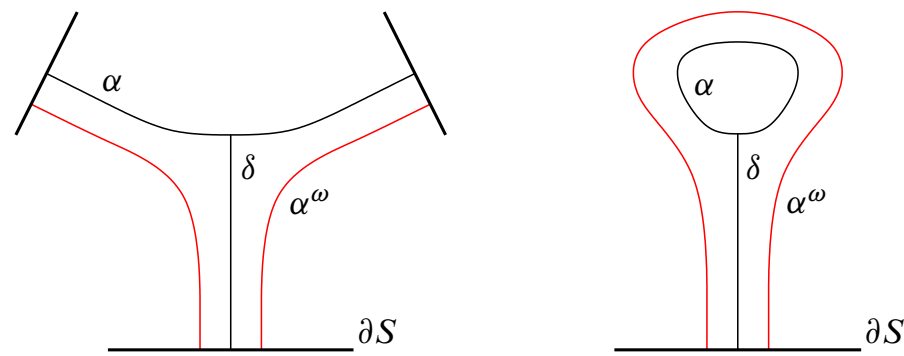

Figure 2.1: The result of surgery, $\alpha^{\omega}$, is either a pair of arcs or a single arc as $\alpha$ is an arc or a curve.

We call the arcs of $\alpha^{\omega}$ the children of $\alpha$. Define $A^{\omega}=(A-\alpha) \cup \alpha^{\omega}$; this is the result of surgering $A$ exactly once along $\omega$.

Lemma 2-4 Suppose $A, B$ are systems and $\omega$ is a directed arc cutting $B$. Then $\left|\operatorname{inner}\left(A^{\omega}, B\right)-\operatorname{inner}(A, B)\right| \leq 1$.

Proof Note that $A^{\omega} \cup A$ is again a system. Two applications of (2-1) give

$$
\begin{aligned}
& \text { inner }\left(A^{\omega} \cup A, B\right) \leq \operatorname{inner}\left(A^{\omega}, B\right) \leq \operatorname{inner}\left(A^{\omega} \cup A, B\right)+1, \\
& \text { inner }\left(A^{\omega} \cup A, B\right) \leq \operatorname{inner}(A, B) \leq \operatorname{inner}\left(A^{\omega} \cup A, B\right)+1 .
\end{aligned}
$$

When $B=\{\omega\}$ a stronger result holds.

Proposition 2-5 Suppose $A$ is a system and $\omega$ is a directed $\operatorname{arc} c u t t i n g ~ A$. Then $\operatorname{inner}\left(A^{\omega}, \omega\right) \leq \operatorname{inner}(A, \omega)$. 
Proof We induct on inner $(A, \omega)$. Suppose that inner $(A, \omega)=n+1$. Let $\alpha$ be the element of $A$ realizing the minimal distance to $\omega$. There are two cases. If $\alpha$ is not the active element then $\alpha \in A^{\omega}$ and the inner distance remains the same or decreases. For example, this occurs when $n=0$.

Suppose, instead, that $\alpha$ is the active element and that $n>0$. Pick $\beta \in \mathcal{A C}(S)$ with:

- $d_{S}(\alpha, \beta)=1$.

- $d_{S}(\beta, \omega)=n$.

- Subject to the above, $\beta$ minimizes $\mathrm{i}(\beta, \omega)$.

Consider the system $B=\{\alpha, \beta\}$. The induction hypothesis gives $\operatorname{inner}\left(B^{\omega}, \omega\right) \leq$ inner $(B, \omega)$. If $\beta$ is the active element of $B$ then we contradict the minimality of $\beta$. Thus $\alpha$ is the active element of $B$. We deduce inner $\left(\alpha^{\omega}, \omega\right) \leq d_{S}(\alpha, \omega)$, completing the proof.

If $A$ is a system and $\omega$ is a directed arc cutting $A$ then we define a surgery sequence starting at $A$ with target the directed arc $\omega$, as follows. Set $A_{0}=A$ and let $A_{i+1}=A_{i}^{\omega}$; that is, we obtain $A_{i+1}$ by surgering the active element of $A_{i}$ exactly once along $\omega$. The arc $\omega$ misses the last system $A_{N}$; the resulting sequence is $\left\{A_{i}\right\}_{i=0}^{N}$.

Given integers $i \leq j$ we adopt the notation $[i, j]=\{k \in \mathbb{Z} \mid i \leq k \leq j\}$.

Lemma 2-6 Suppose $\left\{A_{i}\right\}_{i=0}^{N}$ is a surgery sequence with target $\omega$. Then for each distance $d \in[0$, inner $(A, \omega)-1]$ there is an index $i \in[0, N]$ such that $\operatorname{inner}\left(A, A_{i}\right)=d$.

Proof Since outer $\left(A_{N}, \omega\right) \leq 1$ the triangle inequality

$$
\operatorname{inner}(A, \omega) \leq \operatorname{inner}\left(A, A_{N}\right)+\operatorname{inner}\left(A_{N}, \omega\right)
$$

holds without additive error. Thus $\operatorname{inner}\left(A, A_{N}\right) \geq \operatorname{inner}(A, \omega)-1$. The conclusion now follows from Lemma 2-4.

We can also generalize Proposition 2-5 to sequences. As we do not use this in the remainder of the paper, we omit the proof.

Proposition 2-7 Suppose $\left\{A_{i}\right\}_{i=0}^{N}$ is a surgery sequence with target $\omega$. Let $\alpha_{k} \subset A_{k}$ be the active element and set $\omega_{k}=\alpha_{k}^{\omega}$. Then $\operatorname{inner}\left(A_{i+1}, \omega_{k}\right) \leq \operatorname{inner}\left(A_{i}, \omega_{k}\right)$, for $i<k$. 
Suppose $B \subset A$ is a subsystem and $\omega$ is a directed arc cutting $A$. Let $\left\{A_{i}\right\}$ be the surgery sequence starting at $A$ with target $\omega$. Let $B_{0}=B$ and suppose we have defined $B_{i} \subset A_{i}$. If the active element $\alpha \in A_{i}$ is not in $B_{i}$ then we define $B_{i+1}=B_{i}$. If the active element $\alpha \in A_{i}$ is in $B_{i}$ then define $B_{i+1}=B_{i}^{\omega}$. In any case we say that the elements of $B_{i+1}$ are the children of the elements of $B_{i}$; for $j \geq i$ we say that the elements of $B_{j}$ are the descendants of $B_{i}$. We call the sequence $\left\{B_{i}\right\}$ a surgery sequence with waiting times; the sequence $\left\{B_{i}\right\}$ is subordinate to $\left\{A_{i}\right\}$.

If $A=\{\gamma\}$ is a single arc, and $\omega$ is understood from the context, then we allow ourselves to abuse notation and simply refer to the descendants of $\gamma$.

\section{Descendants}

The goal of this section is to prove Lemma 3-3: disjoint systems have a common descendant within constant distance. Recall that a simplex $A \subset \mathcal{A C}(S)$ is called a system.

Lemma 3-1 Suppose $A$ is a system and $\omega$ is a directed arc cutting $A$. Suppose $\gamma \in \mathcal{C}(S)$ is a curve. If $\gamma$ cuts $A$ then $\gamma$ cuts $A^{\omega}$.

Proof Suppose $\alpha \in A$ is the active element. If $\gamma$ cuts some element of $A-\alpha$ then there is nothing to prove. If $\gamma$ cuts $\alpha$ then, consulting Figure 2.1, the curve $\gamma$ also cuts $\alpha^{\omega}$ and so cuts $A^{\omega}$.

Lemma 3-2 Suppose $\left\{A_{i}\right\}$ is a surgery sequence with target $\omega$. For any index $k$, if $\operatorname{outer}\left(A_{0}, A_{k}\right) \geq 3$ then $A_{j}$ is filling for all $j \geq k$.

Proof By Lemma 3-1 it suffices to prove that $A_{k}$ is filling. Pick any $\gamma \in \mathcal{C}(S)$. Since $\operatorname{outer}\left(A_{0}, A_{k}\right) \geq 3$ it follows that $\gamma$ cuts $A_{0}$ or $A_{k}$, or both. If $\gamma$ cuts $A_{k}$ we are done. If $\gamma$ cuts $A_{0}$ then we are done by Lemma 3-1.

Lemma 3-3 Suppose $A$ is a system and $\omega$ is a directed $\operatorname{arc}$ with $\operatorname{inner}(A, \omega) \geq 6$. Suppose $B, C \subset A$ are subsystems. Let $\left\{A_{i}\right\}_{i=0}^{N}$ be the surgery sequence starting at $A_{0}=A$ with target $\omega$. Let $\left\{B_{i}\right\}$ and $\left\{C_{i}\right\}$ be the subordinate surgery sequences. Then there is an index $k \in[0, N]$ such that:

(1) $B_{k} \cap C_{k} \neq \varnothing$.

(2) inner $\left(A_{0}, A_{i}\right) \leq 5$ for all $i \in[0, k]$.

We paraphrase this as "the subsystems $B$ and $C$ have a common descendant within constant distance of $A$ ". 
Proof of Lemma 3-3 Let $\ell$ be the first index with inner $\left(A, A_{\ell}\right)=3$. Note that $\ell$ exists by Lemma 2-6. Also, Lemma 2-4 implies $\operatorname{inner}\left(A, A_{\ell-1}\right)=2$. Suppose $\beta$ is the active element of $A_{\ell-1}$. Then $\operatorname{inner}(A, \beta)=2$ and $\beta$ is the only element of $A_{\ell-1}$ with this inner distance to $A$. Thus every $\alpha \in A_{\ell}$ has inner distance three to $A$. If $\omega$ misses some element of $A_{\ell}$ then inner $(A, \omega) \leq 4$, contrary to hypothesis. Thus $\omega$ cuts every element of $A_{\ell}$. Isotope the arcs of $A_{\ell}$ to be pairwise disjoint and to intersect $\omega$ minimally.

If $B_{\ell} \cap C_{\ell} \neq \varnothing$ then we take $k=\ell$ and we are done. Suppose instead $B_{\ell}$ and $C_{\ell}$ are disjoint. Since inner $\left(A, A_{\ell}\right)=3$ we have that both $\operatorname{outer}\left(B, B_{\ell}\right)$ and $\operatorname{outer}\left(C, C_{\ell}\right)$ are at least three. Deduce from Lemma 3-2 that $B_{\ell}$ and $C_{\ell}$ both fill $S$, and thus consist only of arcs. Let $B^{\prime} \subset B_{\ell}$ and $C^{\prime} \subset C_{\ell}$ be minimal filling subsystems.

Set $x=-\chi(S)=2 g-2+n$. Set $b=1$ if $S-B^{\prime}$ is a disk. Set $b=0$ if $S-B^{\prime}$ is a union of peripheral annuli. Lemma 2-3 implies $\left|B^{\prime}\right|=x+b$. Define $c$ similarly, with respect to $C^{\prime}$. Let $A^{\prime}=B^{\prime} \cup C^{\prime}$. Let $p$ be the number of peripheral annuli in $S-A^{\prime}$. Observe that if either $b$ or $c$ is one, then $p$ is zero.

We build a graph $G$, dual to $A^{\prime}$, as follows. For every component $R \subset S-A^{\prime}$ there is a dual vertex $v_{R}$. For every $\operatorname{arc} \alpha \in A^{\prime}$ there is a dual edge $e_{\alpha}$; the two ends $e_{\alpha}$ are attached to $v_{R}$ and $v_{Q}$ where $R$ and $Q$ meet the two sides of $\alpha$. Note the possibility that $R$ equals $Q$. Finally, for every peripheral annulus component $P \subset S-A^{\prime}$ there is a peripheral edge $e_{P}$. Both ends of $e_{P}$ are attached to $v_{P}$.

Since $B^{\prime} \cap C^{\prime}=\varnothing,\left|A^{\prime}\right|=\left|B^{\prime}\right|+\left|C^{\prime}\right|$. Thus $G$ has $\left|A^{\prime}\right|+p=2 x+b+c+p$ edges. Since $S$ is homotopy equivalent to $G$, we deduce that $G$ has $x+b+c+p$ vertices. Since $A^{\prime}=B^{\prime} \cup C^{\prime}$ is a filling system, the graph $G$ has no vertices of degree one or two.

Claim One of the following holds.

(1) The graph $G$ has a vertex of degree three, dual to a disk component of $S-A^{\prime}$.

(2) Every vertex of $G$ has degree four and every component of $S-A^{\prime}$ is a disk.

Proof of Claim Let $V_{d}$ denote the number of vertices of $G$ with degree $d$. As there are no vertices of degree one or two, twice the number of edges of $G$ equals $\sum_{d \geq 3} d \cdot V_{d}$. Hence

$$
\begin{aligned}
4 x+2 b+2 c+2 p=\sum_{d \geq 3} d \cdot V_{d} \geq 3 V_{3}+4 \sum_{d \geq 4} V_{d} & =4 \sum_{\begin{array}{l}
d \geq 3 \\
\end{array}} V_{d}-V_{3} \\
& =4 x+4 b+4 c+4 p-V_{3} .
\end{aligned}
$$

Therefore, $V_{3} \geq 2 b+2 c+2 p$ where equality holds if and only if $V_{d}=0$ for $d \geq 5$. If $p=0$ then either $V_{3}>0$, and we obtain the first conclusion, or $V_{3}=0$, and we have the second. If $p>0$ then $V_{3} \geq 2 p$ and we obtain the first conclusion. 
Let $\left\{\delta_{i}\right\}_{i=1}^{M}$ enumerate the arcs of $\omega \cap\left(S-A_{\ell}\right)$, where the order of the indices agrees with the orientation of $\omega$. So the system $A_{\ell+1}$ is obtained from $A_{\ell}$ via surgery along $\delta_{1}$. Generically, our strategy is to find a disk component $R \subset S-A_{\ell}$ and an arc $\delta_{i} \subset R$ so that:

- $\delta_{i}$ meets both $B_{\ell}$ and $C_{\ell}$.

- $\delta_{i}$ is parallel in $R$ to a subarc of $\partial S$.

That is, $\delta_{i}$ cuts a rectangle off of $R$. Surgery along $\delta_{i}$ then produces a common descendant for the systems $B$ and $C$.

Suppose conclusion (1) of the claim holds. Deduce there is a disk component $R \subset$ $S-A_{\ell}$ that is combinatorially a hexagon, with sides alternating between $\partial S$ and $A_{\ell}$. Furthermore, minimality implies $R$ meets both $B_{\ell}$ and $C_{\ell}$. As a very special case, if $\delta_{1}$ lies in $R$ then take $k=\ell+1$ and we are done. See the left-hand side of Figure 3.1.
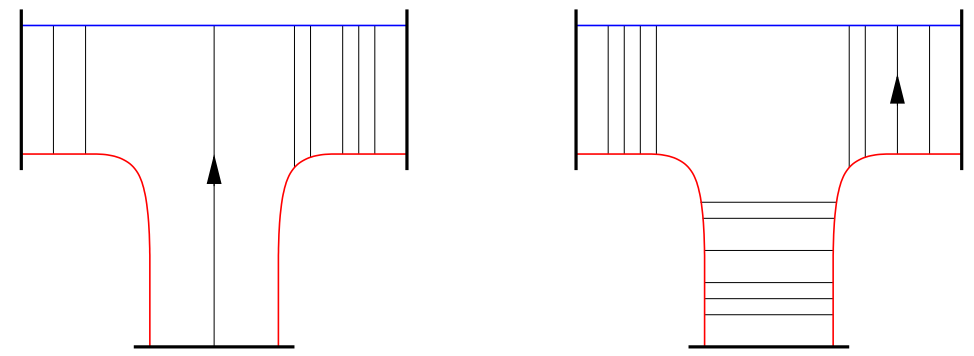

Figure 3.1: The lower and the vertical sides of $R$ lie in $\partial S$ : the longer boundary arcs lie in $A_{\ell}$. The arcs in the interior are subarcs of $\omega$. The arc with the arrow is $\delta_{1}$ on the left and is $\delta_{m}$ on the right.

If $\delta_{1}$ does not lie in $R$, then let $\delta_{m}$ be the first arc contained in $R$ that meets both $B_{\ell}$ and $C_{\ell}$. Set $k=\ell+m$. See the right-hand side of Figure 3.1. Breaking symmetry, two of the three $\operatorname{arcs}$ of $\operatorname{fr}(R)$ lie in $B_{\ell}$. Thus the third arc lies in $C_{\ell}$ and survives to $A_{k-2}$. Thus inner $\left(A, A_{i}\right) \leq 3$ for all $i \in[\ell, k-2]$. The $\operatorname{arcs}$ of $B_{\ell}$ in the frontier of $R$ may be surgered during the interval $[\ell+1, k-2]$, but there is always a hexagon bounded by their children, containing the arc $\delta_{m}$. Surgering $\delta_{m}$ produces the desired common descendants in $A_{k}$. Finally, we note that $\operatorname{inner}\left(A, A_{k-1}\right)$ and $\operatorname{inner}\left(A, A_{k}\right)$ are at most 4 as a child of an $\operatorname{arc}$ of $\operatorname{fr}(R)$ is in both $A_{k-1}$ and $A_{k}$. Hence the lemma holds in this case.

Suppose instead that conclusion (2) of the claim holds. Thus every component of $S-A^{\prime}$ is combinatorially an octagon with sides alternating between $\partial S$ and $A^{\prime}$. If $A_{\ell} \neq A^{\prime}$ then there is an arc of $A_{\ell}-A^{\prime}$ that lies in the interior of one of the octagons. 
Thus $S-A_{\ell}$ has a disk component that is combinatorially a hexagon, and the above argument applies. Therefore, we assume $A^{\prime}, B^{\prime}, C^{\prime}=A_{\ell}, B_{\ell}, C_{\ell}$.

Fix a component $R \subset S-A_{\ell}$ that does not contain $\delta_{1}$. We refer to the four sides of $\operatorname{fr}(R) \subset A_{\ell}$ using the cardinal directions N, S, E and W. Up to interchanging $B_{\ell}$ and $C_{\ell}$, there are three cases to consider, depending on how N, S, E and W lie in $B_{\ell}$ or $C_{\ell}$.

Suppose that $\mathrm{N}$ lies in $B_{\ell}$ and the three other sides lie in $C_{\ell}$. Suppose there is an arc $\delta_{i}$ in $R$ connecting $\mathrm{N}$ to $\mathrm{E}$ or $\mathrm{N}$ to $\mathrm{W}$. Let $\delta_{m}$ be the first such arc. Arguing as before, under conclusion (1), the lemma holds. If there is no such arc, then, as $\omega$ cuts $\mathrm{N}$, there is an $\operatorname{arc} \delta_{i}$ connecting $\mathrm{N}$ to $\mathrm{S}$. Let $\delta_{m}$ be the first such arc; set $k=\ell+m$. As $\mathrm{N} \in A_{j}$ for all $j \in[\ell, k-2]$, deduce $\operatorname{inner}\left(A, A_{i}\right) \leq 3$ for all such $j$. Also $\operatorname{inner}\left(A, A_{k-1}\right)$ and inner $\left(A, A_{k}\right)$ are at most 4 as a child of an $\operatorname{arc}$ of $\operatorname{fr}(R)$ is in both $A_{k-1}$ and $A_{k}$. We now observe that some descendants of $\operatorname{fr}(R)$ cobound a combinatorial hexagon $R^{\prime}$ in $S-A_{k}$. If $\omega$ misses any arc in the frontier of $R^{\prime}$, then $\operatorname{inner}(A, \omega) \leq 5$, contrary to the hypothesis. Else, arguing as in conclusion (1), the lemma holds.

Suppose $\mathrm{N}$ and $\mathrm{E}$ lie in $B_{\ell}$ while $\mathrm{S}$ and $\mathrm{W}$ lie in $C_{\ell}$. If there is an arc connecting $\mathrm{N}$ to $\mathrm{W}$ or connecting $\mathrm{E}$ to $\mathrm{S}$, then surgery along the first such produces common descendants. If there is no such arc, then there must be an arc connecting $\mathrm{N}$ to $\mathrm{S}$ or an arc connecting $\mathrm{E}$ to $\mathrm{W}$; if not $\omega$ misses one of the diagonals of $R$, so $\operatorname{inner}\left(\omega, A_{\ell}\right) \leq 2$ implying inner $(\omega, A) \leq 5$, contrary to assumption. Again, surgery along the first such arc produces a combinatorial hexagon.

Suppose finally that $\mathrm{N}$ and $\mathrm{S}$ lie in $B_{\ell}$ while $\mathrm{E}$ and $\mathrm{W}$ lie in $C_{\ell}$. Surgery along the first arc connecting $B_{\ell}$ to $C_{\ell}$, inside of $R$, produces common descendants. Such an arc exists because $\omega$ cuts every $\operatorname{arc}$ of $A_{\ell}$.

\section{Footprints}

In this section we define the footprint of an arc or curve on a surgery sequence. This is not to be confused with the projection, which is defined in Section 5.

Fix $\gamma \in \mathcal{A C}(S)$. Suppose $A$ is a system and $\omega$ is a directed arc. Let $\left\{A_{i}\right\}_{i=0}^{N}$ be the surgery sequence starting at $A$ with target $\omega$. We define $\phi(\gamma)$, the footprint of $\gamma$ on $\left\{A_{i}\right\}$, to be the set

$$
\phi(\gamma)=\left\{i \in[0, N] \mid \gamma \text { misses } A_{i}\right\} .
$$

Note that if $\gamma$ is an element of $A_{i}$ then $i$ lies in the footprint $\phi(\gamma)$.

Lemma 4-1 With $\gamma, A, \omega$ as above, the footprint $\phi(\gamma)$ is an interval. 
Proof When $\gamma$ is a curve, this follows from Lemma 3-1. So suppose that $\gamma$ is an arc. Without loss of generality we may assume $\phi(\gamma)$ is nonempty and $\min \phi(\gamma)=0$. Note that if $\omega$ misses $\gamma$ then we are done. Isotope $\gamma, A$ and $\omega$ to minimize their intersection numbers.

We now surger $A_{0}=A$. These surgeries are ordered along $\omega$. Let $\alpha_{i}$ be the active element of $A_{i}$. Let $\delta_{i} \subset \omega$ be the surgery arc for $\alpha_{i}$, in other words, the subarc of $\omega$ with endpoints the initial endpoint of $\omega$ and the initial intersection point between $\omega$ and $\alpha_{i}$. We define a pair of intervals by

$$
\begin{aligned}
& I=\left\{i \mid \delta_{i-1} \cap \gamma=\varnothing\right\} \cup\{0\}, \\
& J=\left\{i \mid \delta_{i-1} \cap \gamma \neq \varnothing\right\} .
\end{aligned}
$$

The inclusions $\delta_{i-1} \subset \delta_{i}$ and the fact that $\gamma$ misses $A_{0}$ implies that $I \subset \phi(\gamma)$. To finish the proof we will show $J \cap \phi(\gamma)=\varnothing$, implying that $I=\phi(\gamma)$.

Fix any $k \in J$. Let $\alpha_{k-1}$ be the active element of $A_{k-1}$. As $\alpha_{k-1}$ is an arc or a curve we consult the left- or right-hand side of Figure 2.1. Note that $\gamma$ meets $\delta_{k-1}$, and $\gamma$ is an arc, so it enters and exits the region cobounded by $\alpha_{k-1}$ and its children. Thus $\gamma$ cuts $A_{k}$ and we are done.

\section{Projections to surgery sequences}

Here, in Propositions 5-4 and 5-5, and in Corollary 5-8 we verify that a surgery path has a projection map satisfying three properties, called here the retraction axiom, the Lipschitz axiom and the contraction axiom. These were first set out by Masur and Minsky [14, Definition 2.2]. We closely follow Handel and Mosher [9]. We also refer to the paper of Hilion and Horbez [13]. We emphasize that the various constants appearing in our argument are uniform, that is, independent of the surface $S=S_{g, n}$, mainly by virtue of Lemma 3-3.

The relevance of the three axioms is given by the following theorem of Masur and Minsky [14, Theorem 2.3].

Theorem 5-1 If $\mathcal{X}$ has an almost transitive family of paths, with projections satisfying the three axioms, then $\mathcal{X}^{(1)}$ is hyperbolic. Furthermore, the paths in the family are uniform reparametrized quasigeodesics.

Before turning to definitions, we remark that the hyperbolicity constant and the quasigeodesic constants depend only on the constants coming from almost transitivity and from the three axioms. In Section 6 we provide a proof of Theorem 5-1, giving an estimate for the resulting hyperbolicity constant. 


\subsection{Transitivity}

Suppose that $\mathcal{X}$ is a flag simplicial complex. A path is a sequence $\left\{\sigma_{i}\right\}_{i=0}^{N}$ of simplices in $\mathcal{X}$. A family of paths in $\mathcal{X}$ is $d$-transitive (or simply almost transitive) if for any vertices $x, y \in \mathcal{X}^{(0)}$ there exists a path $\left\{\sigma_{i}\right\}_{i=0}^{N}$ in the family such that inner $\left(x, \sigma_{0}\right)$, inner $\left(\sigma_{i}, \sigma_{i+1}\right)$ and inner $\left(\sigma_{N}, y\right)$ are all at most $d$.

Lemma 5-2 (Transitivity) Surgery sequences form a 2-transitive family of paths.

Proof Fix $\alpha, \beta \in \mathcal{A C}(S)$. Pick an oriented arc $\omega \in \mathcal{A C}(S)$ so that $\mathrm{i}(\beta, \omega)=0$. Let $\left\{A_{i}\right\}_{i=0}^{N}$ be the surgery sequence starting at $A_{0}=\{\alpha\}$ with target $\omega$. Since $\operatorname{inner}\left(A_{N}, \beta\right) \leq 2$, the lemma is proved.

\subsection{Projection}

We now define the projection map to a surgery sequence, following Handel and Mosher; see Figure 1.1. We then state and verify the three axioms in our setting.

Definition 5-3 (Projection) Suppose $\left\{A_{i}\right\}_{i=0}^{N}$ is a surgery sequence with target $\omega$. We define the projection map $\pi: \mathcal{A C}(S) \rightarrow[0, N]$ as follows. Fix $\beta \in \mathcal{A C}(S)$. Suppose that $\left\{B_{j}\right\}$ is the surgery sequence starting at $B=\{\beta\}$ with target $\omega$. Define $\pi(\beta)$ to be the least index $m \in[0, N]$ so that there is an index $k$ with $A_{m} \cap B_{k} \neq \varnothing$. If no such index $m$ exists then we set $\pi(\beta)=N$.

In the following we use the notation $[i, j]=[\min \{i, j\}, \max \{i, j\}]$ when the order is not important. We also write $A[i, j]$ for the union $\bigcup_{k \in[i, j]} A_{k}$.

Proposition 5-4 (Retraction) For any surgery sequence $\left\{A_{i}\right\}_{i=0}^{N}$, index $k \in[0, N]$, and element $\beta \in A_{k}$, the diameter of $A[\pi(\beta), k]$ is at most two.

Proof Let $\left\{B_{j}\right\}_{j=k}^{N}$ be the surgery sequence subordinate to $\left\{A_{i}\right\}_{i=k}^{N}$ that starts at $B=\{\beta\}$. Set $m=\pi(\beta)$; note that $m \leq k$, as $\beta \in B_{k} \subset A_{k}$.

Suppose that $A_{m} \cap B_{\ell} \neq \varnothing$ for some $\ell \geq k$. As $\left\{B_{j}\right\}$ is subordinate to $\left\{A_{i}\right\}$ we have $B_{\ell} \subset A_{\ell}$. Pick any $\gamma \in A_{m} \cap A_{\ell}$. By Lemma 4-1 we have that $[m, \ell]$ lies in $\phi(\gamma)$, the footprint of $\gamma$. Thus $[m, k]$ lies in $\phi(\gamma)$. Thus the diameter of $A[m, k]$ is at most two, finishing the proof.

Instead of using footprints, Hilion and Horbez [13, Proposition 5.1] verify the retraction axiom by using the fact that intersection numbers decrease monotonically along a surgery sequence. 
The verification of the final two axioms is identical to that of Handel and Mosher [9]: replace their Proposition 6.5 in the argument of Section 6.3 with Lemma 3-3. Alternatively, in the geometric setting these arguments appear in [13, Section 7]: replace their Proposition 7.1 with our Lemma 3-3.

Proposition 5-5 (Lipschitz) There is a constant $c$ with the following property: For any surgery sequence $\left\{A_{i}\right\}_{i=0}^{N}$ and any vertices $\beta, \gamma \in \mathcal{A C}(S)$, if $d_{S}(\beta, \gamma) \leq 1$, then the diameter of $A[\pi(\beta), \pi(\gamma)]$ is at most $c$.

In fact, $c=14$ suffices.

Proof Let $m=\pi(\beta)$ and $k=\pi(\gamma)$. Without loss of generality we may assume that $m \leq k$. There are two cases. Suppose that inner $\left(A_{m}, \omega\right) \leq 6$. By Proposition 2-5, for all $i \geq m$ we have $\operatorname{inner}\left(A_{i}, \omega\right) \leq 6$. It follows that the diameter of $A[m, k]$ is at most 14 .

Suppose instead that inner $\left(A_{m}, \omega\right) \geq 7$. Fix some $\beta^{\prime} \in A_{m}$, a descendant of $\beta$. Thus there is a descendant $\gamma^{\prime}$ of $\gamma$ with $d_{S}\left(\beta^{\prime}, \gamma^{\prime}\right) \leq 1$. Set $B^{\prime}=\left\{\beta^{\prime}, \gamma^{\prime}\right\}$ and note that $\operatorname{inner}\left(B^{\prime}, \omega\right) \geq 6$. Let $\left\{B_{i}^{\prime}\right\}$ be the resulting surgery sequence with target $\omega$.

By Lemma 3-3, there is an index $p$ and some $\delta \in B_{p}^{\prime}$ that is a common descendant of both $\beta^{\prime}$ and $\gamma^{\prime}$. Additionally, any vertex of $B^{\prime}[0, p]$ has inner distance to $B^{\prime}=B_{0}^{\prime}$ of at most five. Now, since $\delta$ is a descendant of $\beta^{\prime}$ there is some least index $q$ so that $\delta \in A_{q}$. Thus $k \leq q$. It follows that the diameter of $A[m, k]$ is at most 14 .

Proposition 5-6 (Weak contraction) There are constants $a^{\prime}, b^{\prime}, c$ with the following property: For any surgery sequence $\left\{A_{i}\right\}_{i=0}^{N}$ and any vertices $\beta, \gamma \in \mathcal{A C}(S)$, if

- $\quad \operatorname{inner}(\beta, A[0, N]) \geq a^{\prime}$ and

- $d_{S}(\beta, \gamma) \leq b^{\prime} \cdot \operatorname{inner}(\beta, A[0, N])$,

then the diameter of $A[\pi(\beta), \pi(\gamma)]$ is at most $c$.

In fact, $a^{\prime}=24, b^{\prime}=\frac{1}{8}$ and $c=14$ suffice.

Proof Suppose $\left\{A_{i}\right\}_{i=0}^{N}$ is a surgery sequence with target $\omega$. Let $\pi: \mathcal{A C}(S) \rightarrow[0, N]$ denote the projection to the surgery sequence $\left\{A_{i}\right\}$. Let $\left\{B_{j}\right\}_{j=0}^{M}$ be the surgery sequence starting with $B_{0}=\{\beta\}$ with target $\omega$.

We begin with a sketch of the proof. The contraction axiom is verified by repeatedly applying Lemma 3-3: if two arcs or curves are far from $\left\{A_{i}\right\}_{i=0}^{N}$ but proportionally close to one another, then their surgery sequences have a common descendant prior to intersecting $\left\{A_{i}\right\}$. An application of the Lipschitz axiom, Proposition 5-5, completes the proof. The remainder of Section 5.2 is devoted to supplying the details of this sketch. 
For the purpose of the following claim, we use the following, weaker, hypotheses: inner $(\beta, A[0, N]) \geq 21$ and $d_{S}(\beta, \gamma) \leq \frac{1}{7} \operatorname{inner}(\beta, A[0, N])$. This is necessary because of the asymmetry between $\beta$ and $\gamma$.

Claim There is an index $k \in[0, M]$ so that:

- $B_{k}$ contains a descendant of $\gamma$.

- $\operatorname{inner}\left(\beta, B_{j}\right) \leq 6 d_{S}(\beta, \gamma)$ for all $j \in[0, k]$.

Proof of claim Fix $\alpha \in \mathcal{A C}(S)$ such that $d_{S}(\beta, \alpha)=d_{S}(\beta, \gamma)-1$ and $\mathrm{i}(\alpha, \gamma)=0$. By induction, there is an index $\ell \in[0, M]$ such that $B_{\ell}$ contains a common descendant of $\alpha$ and $\beta$, so that $\operatorname{inner}\left(\beta, B_{j}\right) \leq 6 d_{S}(\beta, \alpha)=6 d_{S}(\beta, \gamma)-6$ for all $j \in[0, \ell]$. Let $\alpha^{\prime} \in B_{\ell}$ be such a descendant. As $\mathrm{i}(\alpha, \gamma)=0$, it follows that $\gamma$ has a descendant, $\gamma^{\prime}$, that misses $\alpha^{\prime}$. If $\gamma^{\prime}$ is also a descendant of $\beta$ then we are done. Suppose not. Let $B^{\prime}=\left\{\alpha^{\prime}, \gamma^{\prime}\right\}$ and let $\left\{B_{i}^{\prime}\right\}$ be the resulting surgery sequence with target $\omega$.

Repeatedly applying the triangle inequality and the hypotheses we have

$$
\begin{aligned}
\operatorname{inner}\left(B^{\prime}, \omega\right) \geq \operatorname{inner}\left(B_{\ell}, \omega\right)-1 & \geq d_{S}(\beta, \omega)-\operatorname{inner}\left(\beta, B_{\ell}\right)-2 \\
& \geq \operatorname{inner}\left(\beta, A_{N}\right)-1-\left(6 d_{S}(\beta, \gamma)-6\right)-2 \\
& \geq \frac{1}{7} \operatorname{inner}\left(\beta, A_{N}\right)+3 \geq 6 .
\end{aligned}
$$

As in the proof of Proposition 5-5, we use Lemma 3-3 to obtain an index $p$ and element $\delta \in B_{p}^{\prime}$, so that $\delta$ is a common descendant of $\alpha^{\prime}$ and $\gamma^{\prime}$. Additionally, any element of $B^{\prime}[0, p]$ has inner distance to $B^{\prime}$ of at most five. Let $k \in[\ell, M]$ be the first index such that $\delta \in B_{k}$.

What is left to show is that for $j \in[\ell, k]$ we have $\operatorname{inner}\left(\beta, B_{j}\right) \leq 6 d_{S}(\beta, \gamma)$; by induction it holds for $j \in[0, \ell]$. Note that for each $j \in[\ell, k]$ the system $B_{j}$ contains a descendant of $\alpha^{\prime}$. Thus inner $\left(B^{\prime}, B_{j}\right)$ is at most five. We have

$$
\begin{aligned}
\operatorname{inner}\left(\beta, B_{j}\right) & \leq \operatorname{inner}\left(\beta, B^{\prime}\right)+\operatorname{inner}\left(B^{\prime}, B_{j}\right)+1 \\
& \leq\left(6 d_{S}(\beta, \gamma)-6\right)+5+1 \leq 6 d_{S}(\beta, \gamma) .
\end{aligned}
$$

This completes the proof of the claim.

We now complete the proof of the proposition. There are two cases. Suppose $\pi(\beta) \leq \pi(\gamma)$ and the weaker hypotheses hold: $\operatorname{inner}(\beta, A[0, N]) \geq 21$ and $d_{S}(\beta, \gamma) \leq$ $\frac{1}{7}$ inner $(\beta, A[0, N])$. Let $k \in[0, M]$ be as in the claim and let $\gamma^{\prime} \in B_{k}$ be a descendant of $\gamma$. Thus we have $\pi(\gamma) \leq \pi\left(\gamma^{\prime}\right)$. Choose $\ell \in[0, N]$ to minimize $\operatorname{inner}\left(\beta, A_{\ell}\right)$. For all $j \in[0, k]$, by the second bullet of the claim we have

$$
\text { inner }\left(\beta, B_{j}\right) \leq 6 d_{S}(\beta, \gamma) \leq \frac{6}{7} \operatorname{inner}\left(\beta, A_{\ell}\right) \leq \operatorname{inner}\left(\beta, A_{\ell}\right)-3 \text {. }
$$


Therefore, we have that $B_{j} \cap A_{i}=\varnothing$ for all $j \in[0, k]$ and $i \in[0, N]$ and so $\beta$ has a descendant $\beta^{\prime} \in B_{k}$ such that $\pi(\beta)=\pi\left(\beta^{\prime}\right)$. Hence $[\pi(\beta), \pi(\gamma)] \subset\left[\pi\left(\beta^{\prime}\right), \pi\left(\gamma^{\prime}\right)\right]$. By Proposition 5-5 as $d_{S}\left(\beta^{\prime}, \gamma^{\prime}\right) \leq 1$, the diameter of $A\left[\pi\left(\beta^{\prime}\right), \pi\left(\gamma^{\prime}\right)\right]$ is at most $c$. Therefore the diameter of $A[\pi(\beta), \pi(\gamma)]$ is also at most $c$.

We now deal with the remaining case. Suppose $\pi(\beta)>\pi(\gamma)$, inner $(\beta, A[0, N]) \geq 24$ and $d_{S}(\beta, \gamma) \leq \frac{1}{8}$ inner $(\beta, A[0, N])$. Here we proceed along the lines of [9, Lemma 3.2]. We find for all $i \in[0, N]$,

$$
\begin{aligned}
\operatorname{inner}\left(\gamma, A_{i}\right) & \geq \operatorname{inner}\left(\beta, A_{i}\right)-d_{S}(\beta, \gamma) \geq \frac{7}{8} \operatorname{inner}\left(\beta, A_{i}\right) \geq 21, \\
d_{S}(\beta, \gamma) & \leq \frac{1}{8} \operatorname{inner}\left(\beta, A_{i}\right) \leq \frac{1}{7} \operatorname{inner}\left(\gamma, A_{i}\right),
\end{aligned}
$$

where the final inequality follows by dividing (5-7) by seven. As $\pi(\gamma) \leq \pi(\beta)$, the above argument now implies that the diameter of $A[\pi(\beta), \pi(\gamma)]$ is at most $c$.

Corollary 5-8 (Contraction) There are constants $a, b, c$ with the following property: For any surgery sequence $\left\{A_{i}\right\}_{i=0}^{N}$ and any vertices $\beta, \gamma \in \mathcal{A C}(S)$, if

- $\operatorname{inner}\left(\beta, A_{\pi(\beta)}\right) \geq a$ and

- $d_{S}(\beta, \gamma) \leq b \cdot \operatorname{inner}\left(\beta, A_{\pi(\beta)}\right)$,

then the diameter of $A[\pi(\beta), \pi(\gamma)]$ is at most $c$.

In fact, $a=15 a^{\prime}+2, b=b^{\prime} / 16$ and $c=14$ suffice.

Proof Let $\rho \in A[0, N]$ be any closest point of $A[0, N]$ to $\beta$. Set $r=d_{S}(\beta, \rho)$. Note that $r=\operatorname{inner}(\beta, A[0, N])$ is quantity appearing in the statement of Proposition 5-6.

From Proposition 5-5 deduce $\operatorname{diam}(A[\pi(\beta), \pi(\rho)]) \leq c$. From Proposition 5-4 deduce inner $\left(\rho, A_{\pi(\rho)}\right) \leq 2$. Thus, by hypothesis and by the triangle inequality we have

$$
a \leq \operatorname{inner}\left(\beta, A_{\pi(\beta)}\right) \leq 15 r+2 .
$$

Thus $a^{\prime} \leq r$ and we have obtained the first hypothesis of Proposition 5-6.

Since $r>2$ we find that $15 r+2 \leq 16 r$. Since $d_{S}(\beta, \gamma) \leq b \cdot \operatorname{inner}\left(\beta, A_{\pi(\beta)}\right)$ to obtain the second hypothesis of Proposition 5-6 it suffices to bound the latter. By the above we have

$$
b \cdot \operatorname{inner}\left(\beta, A_{\pi(\beta)}\right) \leq b(15 r+2) \leq 16 b r \leq b^{\prime} r
$$

as desired. The conclusion now follows directly from Proposition 5-6. 


\section{Hyperbolicity}

In this section, we use the contraction properties of $\mathcal{A C}^{(1)}(S)$ to prove it is Gromov hyperbolic. This is already proven in [14]. However, we need an explicit estimate for the hyperbolicity constant. Hence, we reproduce the argument here, keeping careful track of constants.

We say a path $g: I \rightarrow \mathcal{X}$ is $(\ell, L)$-Lipschitz if

$$
\frac{|s-t|}{\ell} \leq d_{\mathcal{X}}(g(s), g(t)) \leq L|s-t| .
$$

Let $a, b$ and $c$ be the constants from Corollary 5-8.

Proposition 6-1 Suppose $g:[0, M] \rightarrow \mathcal{A C}^{(1)}(S)$ is $(\ell, L)-$ Lipschitz and $\left\{A_{i}\right\}_{i=0}^{N}$ is a surgery sequence with

$$
\operatorname{inner}\left(g(0), A_{0}\right), \text { inner }\left(g(M), A_{N}\right) \leq 2 .
$$

If $2 c \ell L \geq a b$ then, for every $t \in[0, M]$,

$$
\operatorname{inner}\left(g(t), A_{\pi(g(t))}\right) \leq 4 c \ell L \frac{\ell L+1}{b}+c\left(2 \ell L^{2}+\ell L+2 L+3 / 2\right)+L .
$$

Remark 6-2 Note that the hypothesis $2 c \ell L \geq a b$ holds for the constants $a, b$ and $c$ given by Corollary 5-8 if $\ell, L \geq 1$.

Proof of Proposition 6-1 Define $r(t)=\operatorname{inner}\left(g(t), A_{\pi(g(t))}\right)$. Define

$$
D=\frac{2 c \ell L}{b} \text {. }
$$

Note that $D \geq a$. Let $I=[i, j] \subset[0, M]$ be an interval so that for $t \in I$, we have $r(t) \geq D$ while $r(i-1) \leq D$ and $r(j+1) \leq D$. Set $|I|=|j-i|$. Divide $I$ to intervals of length at most $b D / L$. Assume there are $m$ such intervals and $m$ is minimal; therefore

$$
(m-1) \frac{b D}{L} \leq|I| \leq m \frac{b D}{L} .
$$

Suppose that $J$ is one of these subintervals. By construction $r(t) \geq D \geq a$ for all $t \in J$. Also, note that $g(J)$ has diameter at most $b D$. Hence, Corollary 5-8 applies:

$$
\operatorname{diam}(A[\pi(g(\min J)), \pi(g(\max J))]) \leq c .
$$

Set $R=\max _{t \in I} r(t)$. Since $g(i-1)$ and $g(j+1)$ are within distance $D$ of their surgery projections to $\left\{A_{i}\right\}$ we have

$$
R \leq L \frac{|I|+1}{2}+L+D+c L+c \frac{m+1}{2} .
$$


Also, since $g$ is an $(\ell, L)$-Lipschitz, the distance between $g(i-1)$ and $g(j+1)$ is at least $(|I|+2) / \ell$. Using the lower bound on $|I|$, and the upper bound on diameter (6-4), we deduce

$$
(m-1) \frac{b D}{\ell L} \leq \frac{|I|+2}{\ell} \leq 2 D+2 c L+c m .
$$

Thus

$$
m(b D-c \ell L) \leq 2 \ell D L+b D+2 c \ell L^{2} \Longrightarrow m \leq \frac{4 \ell L}{b}+2 L+2
$$

using (6-3). Rewriting the upper bound on $R$ we find

$$
R \leq \frac{\ell L(2 D+2 c L+c m)}{2}+L+D+c L+\frac{m+1}{2} c .
$$

Again using (6-3) we get

$$
R \leq 4 c \ell L \frac{\ell L+1}{b}+c\left(2 \ell L^{2}+\ell L+2 L+3 / 2\right)+L
$$

as claimed.

Theorem 6-5 If $3 g-3+n \geq 2$ and $n>0$, then $\mathcal{A C}^{(1)}\left(S_{g, n}\right)$ is $\delta$-hyperbolic, where

$$
\delta=\frac{56 c}{b}+\frac{39 c}{2}+2 .
$$

Proof Consider three points $\alpha, \beta, \gamma \in \mathcal{A C}^{(1)}\left(S_{g, n}\right)$. Choose a geodesic segment connecting $\alpha$ to $\beta$ and denote it by $[\alpha, \beta]$. Let $[\beta, \gamma]$ and $[\alpha, \gamma]$ be similarly defined. We need to show that the geodesic segment $[\beta, \gamma]$ is contained in a $\delta$-neighborhood of $[\alpha, \beta] \cup[\alpha, \gamma]$.

Let $\alpha^{\prime}$ be a closest point in $[\beta, \gamma]$ to $\alpha$. The path $p_{\alpha, \beta}$ obtained from the concatenation $\left[\alpha, \alpha^{\prime}\right] \cup\left[\alpha^{\prime}, \beta\right]$ is a $(3,1)$-Lipschitz path [14, page 147]. Since surgery paths are 2 -transitive, we may choose a surgery path $\left\{A_{i}\right\}$ that starts and ends within distance two of $\alpha$ and $\beta$ respectively. With $R$ as in the proof of Proposition 6-1,

$$
\text { if } \ell=3, L=1, \quad \text { then } R \leq \frac{48 c}{b}+\frac{25 c}{2}+1 .
$$

That is, every point in $p_{\alpha, \beta}$ has inner distance at most $48 c / b+25 c / 2+1$ with its projection to $\left\{A_{i}\right\}$. Also by Proposition 6-1,

$$
\text { if } \ell=L=1, \quad \text { then } R \leq \frac{8 c}{b}+\frac{13 c}{2}+1 \text {. }
$$

That is, each point of the geodesic $[\alpha, \beta]$, which is a $(1,1)-$ Lipschitz path, has inner distance at most $8 c / b+13 c / 2+1$ with its projection to $\left\{A_{i}\right\}$. 
By Proposition 5-5, the projection of $[\alpha, \beta]$ to $\left\{A_{i}\right\}$ is $c$-dense. Therefore, any point in $\left\{A_{i}\right\}$ is at most $8 c / b+7 c+1$ away from a point in $[\alpha, \beta]$. Therefore, the path $p_{\alpha, \beta}$ is contained in a

$$
\delta=\frac{56 c}{b}+\frac{39 c}{2}+2
$$

neighborhood of $[\alpha, \beta]$. Similarly, the path $p_{\alpha, \gamma}$ is contained in a $\delta$-neighborhood of $[\alpha, \gamma]$. Hence, $[\beta, \gamma]$ is contained in a $\delta$-neighborhood of $[\alpha, \beta] \cup[\alpha, \gamma]$. That is, $\mathcal{A C}^{(1)}(S)$ is $\delta$-hyperbolic.

\section{Inclusions}

In this section, we show that the hyperbolicity of the curve complex follows from the hyperbolicity of the arc and curve complex.

Corollary 7-1 There is a constant $\mathrm{U}$ such that if $3 g-3+n \geq 2$ and $n>0$ then $\mathcal{C}^{(1)}\left(S_{g, n}\right)$ is $\mathrm{U}$-hyperbolic.

Proof We define the surgery relation $\sigma: \mathcal{A C} \rightarrow \mathcal{C}$ as follows. Fix $\alpha \in \mathcal{A C}$. Let $\delta \subset \partial S$ be the union of the boundary components that $\alpha$ meets. Let $N$ be a regular neighborhood of $\alpha \cup \delta$. Define $\sigma(\alpha)$ to be the frontier of $N$. For $\alpha, \beta \in \mathcal{A C}$ we have

$$
\operatorname{outer}_{\mathcal{C}}(\sigma(\alpha), \sigma(\beta)) \leq 2 d_{\mathcal{A C}}(\alpha, \beta)
$$

by $[15$, Lemma 2.2]. On the other hand, for $\alpha, \beta \in \mathcal{C}$ we have

$$
d_{\mathcal{A C}}(\alpha, \beta) \leq d_{\mathcal{C}}(\alpha, \beta)
$$

Thus the inclusion of $\mathcal{C}^{(1)}\left(S_{g, n}\right)$ into $\mathcal{A C}^{(1)}\left(S_{g, n}\right)$ sends geodesics to $(1,2)$-Lipschitz paths. Continuing as in the proof of Theorem 6-5, we get that the image of a geodesic in $\mathcal{C}$ is in a uniformly bounded neighborhood of a geodesic in $\mathcal{A C}$. Hence, the hyperbolicity of $\mathcal{A C}$ implies the hyperbolicity of $\mathcal{C}$.

We now deal with the case when $S=S_{g}$ is closed.

Theorem 7-2 If $3 g-3 \geq 2$ then $\mathcal{C}^{(1)}\left(S_{g}\right)$ is Gromov hyperbolic. Furthermore, the constant of hyperbolicity is at most that of $\mathcal{C}^{(1)}\left(S_{g, 1}\right)$.

Proof Let $\Sigma=S_{g, 1}$. By Corollary 7-1 we have $\mathcal{C}^{(1)}(\Sigma)$ is U-hyperbolic. Using a construction of Harer [10, Lemma 3.6] (see also the second and third authors [20, Theorem 1.2]), the curve complex $\mathcal{C}^{(1)}(S)$ isometrically embeds in the curve complex $\mathcal{C}^{(1)}(\Sigma)$. 


\section{References}

[1] T Aougab, Uniform hyperbolicity of the graphs of curves, Geom. Topol. 17 (2013) 2855-2875 MR3190300

[2] J Behrstock, B Kleiner, Y N Minsky, L Mosher, Geometry and rigidity of mapping class groups, Geom. Topol. 16 (2012) 781-888 MR2928983

[3] M Bestvina, M Feighn, Hyperbolicity of the complex of free factors, Adv. Math. 256 (2014) 104-155 MR3177291

[4] B H Bowditch, Intersection numbers and the hyperbolicity of the curve complex, J. Reine Angew. Math. 598 (2006) 105-129 MR2270568

[5] B H Bowditch, Uniform hyperbolicity of the curve graphs, Pacific J. Math. 269 (2014) 269-280 MR3238474

[6] J F Brock, R D Canary, Y N Minsky, The classification of Kleinian surface groups, II: The ending lamination conjecture, Ann. of Math. 176 (2012) 1-149 MR2925381

[7] J F Brock, HA Masur, Y N Minsky, Asymptotics of Weil-Petersson geodesic, I: Ending laminations, recurrence and flows, Geom. Funct. Anal. 19 (2010) 1229-1257 MR2585573

[8] U Hamenstädt, Geometry of the complex of curves and of Teichmüller space, from: "Handbook of Teichmüller theory, Vol. I", (A Papadopoulos, editor), IRMA Lect. Math. Theor. Phys. 11, Eur. Math. Soc., Zürich (2007) 447-467 MR2349677

[9] M Handel, L Mosher, The free splitting complex of a free group, I: Hyperbolicity, Geom. Topol. 17 (2013) 1581-1672 MR3073931

[10] J L Harer, The virtual cohomological dimension of the mapping class group of an orientable surface, Invent. Math. 84 (1986) 157-176 MR830043

[11] A Hatcher, On triangulations of surfaces, Topology Appl. 40 (1991) 189-194 MR1123262

[12] S Hensel, P Przytycki, R C H Webb, Slim unicorns and uniform hyperbolicity for arc graphs and curve graphs arXiv:1301.5577

[13] A Hilion, C Horbez, The hyperbolicity of the sphere complex via surgery paths arXiv:1210.6183

[14] H A Masur, Y N Minsky, Geometry of the complex of curves, I: Hyperbolicity, Invent. Math. 138 (1999) 103-149 MR1714338

[15] H A Masur, Y N Minsky, Geometry of the complex of curves, II: Hierarchical structure, Geom. Funct. Anal. 10 (2000) 902-974 MR1791145

[16] Y N Minsky, The classification of punctured-torus groups, Ann. of Math. 149 (1999) 559-626 MR1689341

[17] Y N Minsky, The classification of Kleinian surface groups, I: Models and bounds, Ann. of Math. 171 (2010) 1-107 MR2630036 
[18] K Rafi, Hyperbolicity in Teichmüller space arXiv:1011.6004

[19] K Rafi, A characterization of short curves of a Teichmüller geodesic, Geom. Topol. 9 (2005) 179-202 MR2115672

[20] K Rafi, S Schleimer, Covers and the curve complex, Geom. Topol. 13 (2009) 21412162 MR2507116

Department of Mathematical Sciences, University of Arkansas

SCEN 309, Fayetteville, AR 72701, USA

Department of Mathematics, University of Toronto

Room 6290, 40 St. George Street, Toronto, Ontario M5S 2E4, Canada

Mathematics Institute, University of Warwick

Zeeman Building, Conventry CV4 7AL, UK

mattclay@uark.edu, rafi@math.toronto.edu, s.schleimer@warwick.ac.uk http://comp.uark.edu/ mattclay, http://www.math.toronto.edu/ rafi/, http://homepages.warwick.ac.uk/ masgar/

Received: 16 July 2013 Revised: 24 April 2014 